\title{
An experimental study implementing model reference active structural acoustic control
}

\author{
Robert L. Clark, Gary P. Gibbs, and Chris R. Fuller \\ Mechanical Engineering Department, Virginia Polytechnic Institute and State University, Blacksburg, \\ Virginia 24061
}

(Received 21 May 1992; revised 1 January 1993; accepted 23 February 1993)

\begin{abstract}
Model reference active structural acoustic control is experimentally investigated in this study for physical systems characterized by stationary, narrow-band disturbances in which the acoustic field remains relatively unchanged. The filtered-x version of the multichannel adaptive least-mean-square (LMS) algorithm was implemented on a TMS320C25 digital signal processing board to achieve the desired control approach, and control inputs were generated with piezoelectric actuators. Model reference control provides the designer with a method of replacing acoustic error sensors such as microphones located in the far-field with error sensors such as accelerometers located on the surface of the structure. As opposed to driving the response of the structure to zero at the coordinates of the accelerometers, the response is driven to some predetermined "reference" value corresponding to the desired far-field acoustic directivity pattern. In essence, the uncontrolled structure is adaptively modified to behave like the reference structure under acoustic control conditions. Results from this study indicate that the same acoustic directivity pattern can be achieved with model reference control, and the only requirement is that the number of structural sensors used in the control approach is at least equal to the number of control actuators required to achieve the desired acoustic directivity pattern.
\end{abstract}

PACS numbers: $43.40 . \mathrm{Vn}, 43.50 . \mathrm{Ki}$

\section{INTRODUCTION}

The purpose of this work is to evaluate experimentally the advantages afforded with model reference control for minimizing far-field sound radiation from vibrating structures. Since the primary objective in active structural acoustic control is to minimize far-field sound radiation, microphones located in the acoustic far-field are typically implemented as error sensors in the control approach. However, with the push toward developing adaptive structures configured with control actuators and error sensors embedded within or bonded to the structure, alternative methods of measuring or predicting sound radiation are required. A variety of attempts have been made to develop structural sensors which yield an equivalent cost function to that achieved with sensors located in the acoustic field. In past studies by Clark and Fuller, polyvinylidene fluoride (PVDF) sensors have been optimally shaped and positioned on the surface of structures to provide the appropriate error information for suppressing far-field sound radiation. ${ }^{1-3}$ In addition, a design approach for developing PVDF sensors which weights independent modes of a structure has been outlined such that directional acoustic control can be achieved by selectively suppressing sound radiation at desired far-field coordinates corresponding to specific spectral lines of the wave-number transform. ${ }^{4}$ Preliminary experimental studies have also been devoted to developing error sensors from optical fibers placed on the surface of the structure such that vibration of modes exhibiting the greatest radiation efficiency is suppressed. ${ }^{5,6}$
While all of the above approaches result in significant attenuation of far-field sound radiation, none of the previously outlined sensing techniques yield acoustic control which is identical to that achieved with microphone error sensors, with the exception of PVDF sensors designed to weight independent structural modes. ${ }^{4}$ However at this stage, practical techniques for implementing modally weighted PVDF sensors are not available for complex structures. As an alternative approach for implementing structural error sensors on complex structures, model reference control was investigate analytically by Clark and Fuller. ${ }^{7}$ Results from this study suggest that accelerometers can be located on the structural surface, and as opposed to driving the structural response to zero at these locations, the response is driven to some predetermined magnitude and phase reference value for single frequency or broadband control. In the single frequency case (studied here), a simple finite impulse response filter can be used to create the desired response while in the multifrequency or broadband case, an infinite impulse response filter can be implemented, providing a reference signal correlated with the input disturbance is available. This predetermined structural response corresponds to that resulting in the desired acoustic directivity pattern. The only requirement is that the number of error sensors is at least equal to the number of control actuators necessary to achieve acoustic control. ${ }^{7}$ In other words, if the desired acoustic directivity pattern can be achieved with only one control actuator, then only one error sensor is required in the model reference control approach since the frequency response func- 
tion between that control actuator and structural sensor is unique. The reader is referred to Ref. 7 for a more detailed discussion and proof of this concept.

In this study, experiments were conducted to evaluate and confirm the potential of the model reference control approach for active structural acoustic control. To achieve control, the filtered-x version of the multichannel adaptive LMS algorithm was implemented on a TMS320C25 digital signal processing board resident in an AT-compatible computer. The structure of the LMS algorithm is ideally suited for model reference control since the error to be minimized can readily be defined as the difference between some "desired" plant response and the measured plant response. When the measured response is equivalent in both phase and magnitude to the desired response, the mean square error is zero, and model reference control is achieved. For the purpose of this study, two structures were used to test the controller. Structures with boundary conditions approximating a simply supported beam and a simply supported plate were instrumented with accelerometer error sensors and piezoelectric control actuators. Harmonic excitation of the structures was generated with a shaker to create the input disturbance. Microphones were located in the acoustic field to provide error information as well as evaluate the success of the model reference controller.

When implementing model reference control, a model of the plant is required to compute the desired structural response. As opposed to generating the plant model analytically, the model was obtained experimentally. To develop this model, the controller was initially used to minimize the sound radiation from the structure with microphone error sensors. Upon achieving control, the phase referenced structural response was measured with accelerometers at a number of structural coordinates equal to the number of control actuators. This measurement was performed by conducting a system identification between the reference input (correlated with the input disturbance) to the controller and the response of each accelerometer. The control algorithm was then executed to minimize the difference between the desired response and the measured response at chosen accelerometer coordinates. The result was equivalent attenuation in sound radiation with very similar acoustic directivity patterns. By developing the plant model experimentally, complex structures which cannot readily be modeled analytically can be configured with structural sensors and thereby eliminate the need for microphones located in the radiated acoustic field except when measuring the original system model.

\section{MODEL REFERENCE CONTROLLER}

Before proceeding with the details of the control approach, a brief overview of the model reference controller is in order. A conceptual schematic of a feed-forward adaptive model reference controller is presented in Fig. 1(a). The adaptive plant encompasses the structure, control actuators, error sensors, and control dynamics associated with the multichannel version of the adaptive LMS algorithm, depicted in Fig. 1(b). The reference plant consists of the models required to create the desired response at the

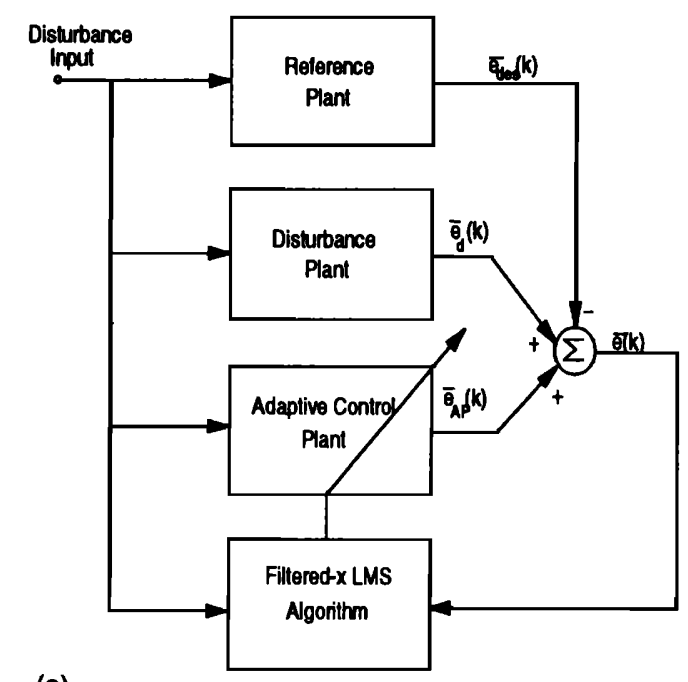

(a)

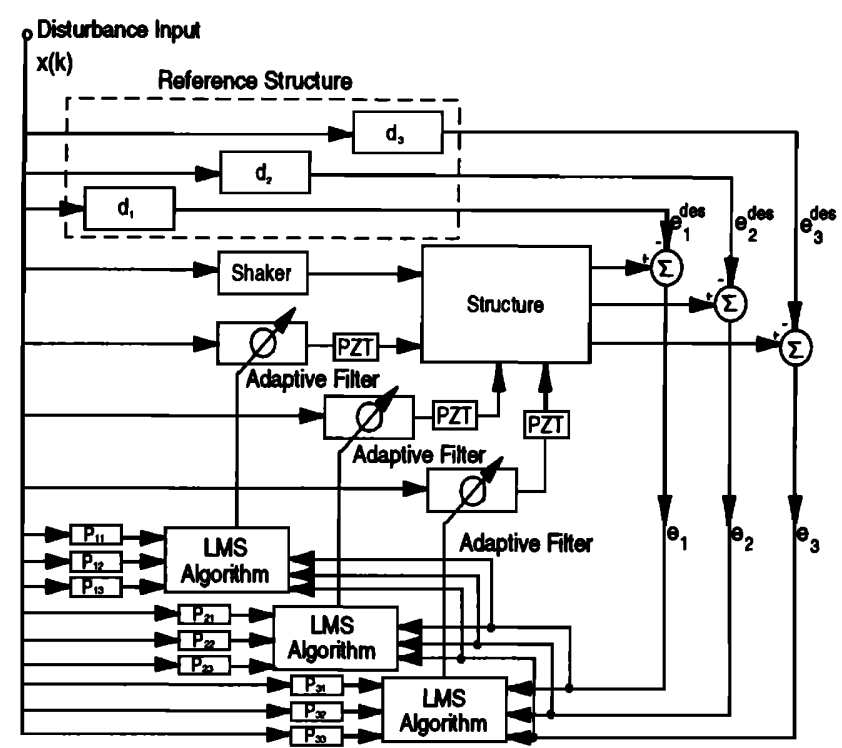

(b)

FIG. 1. LMS model reference controller schematic. (a) Conceptual schematic of model reference controller. (b) Detailed schematic of model reference controller.

chosen error sensor locations, and these models can be obtained analytically or experimentally depending on the designer's choice. The adaptive plant essentially modifies its output until the total error $\bar{e}(k)$, which is defined to be the difference between the desired response, $\bar{e}_{\text {des }}(k)$, and the sum of the adaptive response $\bar{e}_{\mathrm{AP}}(k)$, and the disturbance, $\bar{e}_{\mathrm{d}}(k)$, is minimized.

The method of control chosen for practical implementation in this study is the multichannel filtered-x version of the adaptive LMS algorithm. The formulation of this algorithm readily lends itself to model reference control since the disturbance is included in the output of each error sensor. The multichannel version of this algorithm was previously described by Elliot et al. ${ }^{8}$ and will be briefly reviewed for the reader. The output of an error sensor can be modeled at the $n$th time step as 


$$
\begin{aligned}
e_{l}(n)= & e_{l}^{D}(n)+e_{l}^{\operatorname{des}}(n)+\sum_{m=1}^{M} \sum_{j=0}^{N_{p}-1} P_{l m j} \sum_{i=0}^{N_{w}-1} w_{m i}(n-j) \\
& \times x(n-i-j),
\end{aligned}
$$

where $n=$ time step number; $m=$ actuator number; $l=$ error sensor number; $i=$ adaptive filter coefficient number; $j=$ fixed filter coefficient number; $e_{l}(n)=$ total error the $l$ th error sensor; $e_{l}^{D}(n)=$ error due to the disturbance at the $l$ th error sensor; $e_{l}^{\text {des }}(n)=$ desired response at the $l$ th error sensor; $x(n)=$ input reference source; $w_{m i}$ $=$ coefficients of the adaptive finite impulse response (FIR) filter for the $m$ th actuator and the $i$ th coefficient; $P_{l m j}=j$ th coefficients of the fixed finite impulse response (FIR) filter between the output of the $m$ th adaptive filter and the $l$ th error sensor; $M=$ number of control actuators; $N_{p}=$ number of fixed filter coefficients $\left(N_{p}=2\right.$ here); $N_{w}$ $=$ number of adaptive filter coefficients $\left(N_{w}=2\right.$ here $)$. In the LMS algorithm, the mean square error signal is defined by

$$
J=E\left(\sum_{l=1}^{L} e_{l}^{2}(n)\right)
$$

where $E$ is the expectation operator and $L$ is the number of error sensors. Since this error function is quadratic, only one minimum solution exists. The outputs of the fixed filters $\boldsymbol{P}_{l m j}$ at each time step $n$, were used by the LMS algorithm to minimize the mean square error signal by modifying the coefficients of the adaptive filter as follows

$$
w_{m i}(n+1)=w_{m i}(n)-\mu \sum_{l=1}^{L} e_{l}(n) r_{l m}(n-i),
$$

where

$$
r_{l m}(n-i)=\sum_{j=0}^{N-1} P_{l m j} x(n-i-j)
$$

and $\mu$ is the convergence parameter for the algorithm. Upon achieving control, the adaptive finite impulse response filter coefficients converge to a steady-state value. In typical applications, these coefficients, when multiplied by the reference signal, yield a response equal in magnitude and opposite in phase to the disturbance present (when the number of independent actuators equals the number of error sensors), driving the response at the chosen error sensor to some minimum value. For the case of model reference control, the desired response at the $l$ th error sensor is no longer zero, but rather some predetermined system response.

To implement the equations modeling the error sensors and actuators in the LMS algorithm, the weighting coefficients in the equations for the response of the error sensors must be determined. A schematic of the control approach is presented in Fig. 1(b). The coefficients $P_{l m j}$ define the transfer functions between the control actuators and the error sensors chosen. The desired response for model reference control is generated from an FIR filter with coefficients defined by $d_{l}$, as depicted in Fig. 1(b). ${ }^{9}$ For example, the output of the filter $d_{1}$ can be computed as follows

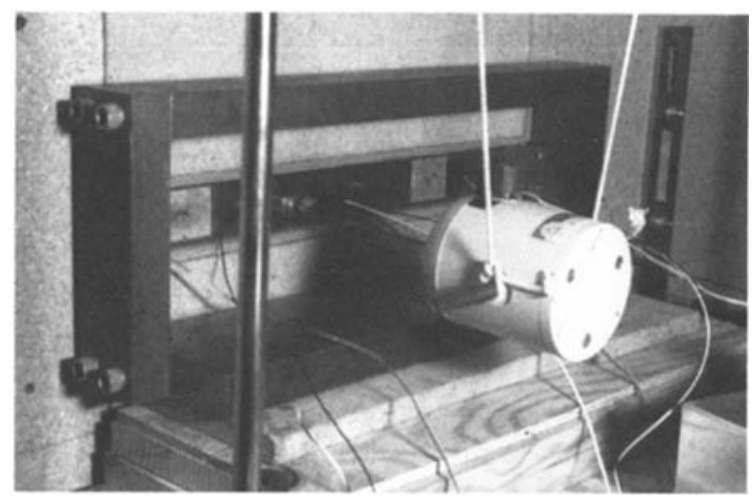

FIG. 2. Simply supported beam/baffle system (back of baffle).

$$
e_{1}^{\text {des }}(k)=d_{1_{0}} x(k)+d_{1_{1}} x(k-1) .
$$

If the desired response at each error sensor is zero, the coefficients defined by $d_{l}$ are simply set equal to zero. As the adaptive coefficients begin to converge, the error signal is driven to a minimum, and hence the response is driven to the desired value at each error sensor. Upon converging, the adaptive coefficients $w_{m i}$ contain the necessary information for computing the optimal control voltage to each actuator.

\section{BEAM AND PLATE CONFIGURATION}

For the purpose of this study, the simply supported beam and simply supported plate were independently positioned in a finite rigid baffle located in the anechoic chamber at VPI and SU where acoustic tests were conducted. Both structures were constructed from cold-rolled steel and the beam measured $380 \mathrm{~mm}$ long $\times 40 \mathrm{~mm}$ wide $\times 4.57$ $\mathrm{mm}$ thick while the plate measured $380 \mathrm{~mm}$ long $\times 300$ $\mathrm{mm}$ wide $\times 1.96 \mathrm{~mm}$ thick. A picture of the beam configured with two piezoelectric actuators and the disturbance shaker is presented in Fig. 2. The piezoelectric actuator used in this experiment is positioned $74 \mathrm{~mm}$ from the right side of the beam as illustrated and measured $38.1 \mathrm{~mm}$ long $\times 31 \mathrm{~mm}$ wide $\times 0.19 \mathrm{~mm}$ thick. The input disturbance was created with a shaker positioned $240 \mathrm{~mm}$ from the right side of the beam. The view point illustrated is from the back side of the rigid baffle.

A picture of the plate is presented in Fig. 3 and four piezoelectric control actuators are depicted. The view point is from the front side of the baffle in the anechoic chamber, and the traversing microphone and boom used to obtain the directivity pattern at $1.6 \mathrm{~m}$ from the horizontal centerline of the plate are illustrated. The three control actuators used in this study were centered at coordinates of $(63,155)$ $\mathrm{mm},(190,250) \mathrm{mm}$, and $(316,50) \mathrm{mm}$ from the lower-left corner of the plate, respectively. Each piezoceramic patch measured $38.1 \mathrm{~mm}$ long $\times 31 \mathrm{~mm}$ wide $\times 0.19 \mathrm{~mm}$ thick, and the full actuator was constructed by placing a patch on the back of the structure with the same spatial coordinates and wiring it electrically out of phase with the front patch. This has previously been shown to result in uniform bending about the neutral axis of the beam upon applying a 


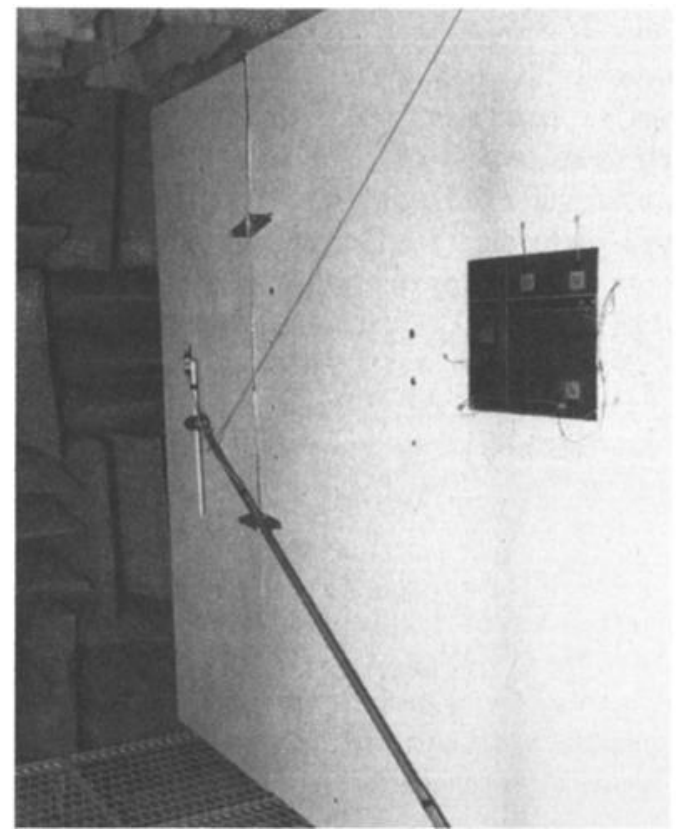

FIG. 3. Simply supported plate/baffle system (front of baffle).

voltage to the actuator patch pair. ${ }^{10,11}$ The shaker was located on the back of the plate at coordinates of $(240,130)$ $\mathrm{mm}$ from the lower-left corner of the front side of the plate (i.e., reference frame of measurement). Also shown are PVDF sensors which were not used in these experiments.

The simply supported boundary conditions for each structure were achieved by attaching the beam or plate to thin shim stock steel such that resistance to rotational motion was small compared to resistance to rigid body motion of the structure. This technique has been successfully used and discussed in previous studies. ${ }^{9,12}$ The resonant frequencies of the first 8 modes of the beam and plate are compared to the predicted resonant frequencies in Table $I$ and Table II, respectively. As indicated from the results, the measured resonant frequencies are within $3 \%$ of the predicted values for the worst case. The mode shapes have also been observed consistent with the predicted shapes in previous experimental studies with test structures approximating simply supported boundary conditions. ${ }^{12}$ Hence, while perfect simply supported boundary conditions cannot be achieved, boundary conditions obtained with the previously outlined technique are close enough to the desired

TABLE I. Theoretical and measured beam resonant frequencies, $f_{m}$.

\begin{tabular}{ccc}
\hline \hline $\begin{array}{c}\text { Mode } \\
(\mathrm{m})\end{array}$ & $\begin{array}{c}\text { Predicted frequency } \\
(\mathrm{Hz})\end{array}$ & $\begin{array}{c}\text { Measured frequency } \\
(\mathrm{Hz})\end{array}$ \\
\hline$(1)$ & 76 & 78 \\
$(2)$ & 303 & 304 \\
$(3)$ & 681 & 680 \\
$(4)$ & 1210 & 1208 \\
$(5)$ & 1891 & 1880 \\
$(6)$ & 2724 & 2704 \\
$(7)$ & 3707 & 3664 \\
$(8)$ & 4841 & 4752 \\
\hline \hline
\end{tabular}

TABLE II. Theoretical and measured plate resonant frequencies, $f_{m n}$.

\begin{tabular}{ccc}
\hline \hline $\begin{array}{c}\text { Mode } \\
(\mathbf{m}, \mathbf{n})\end{array}$ & $\begin{array}{c}\text { Predicted frequency } \\
(\mathbf{H z})\end{array}$ & $\begin{array}{c}\text { Measured frequency } \\
(\mathbf{H z})\end{array}$ \\
\hline$(1,1)$ & 86 & 85 \\
$(2,1)$ & 185 & 183 \\
$(1,2)$ & 245 & 247 \\
$(2,2)$ & 344 & 342 \\
$(3,1)$ & 350 & 351 \\
$(3,2)$ & 508 & 504 \\
$(1,3)$ & 509 & 518 \\
$(4,1)$ & 581 & 603 \\
\hline \hline
\end{tabular}

simply supported boundary conditions that both the structural and acoustic response of the structure can be modeled analytically. Other methods for obtaining simply supported boundary conditions have been discussed as well, and the reader is referred to Ref. 13 for greater detail.

\section{RESULTS}

Control experiments conducted with the simply supported beam are outlined first using a single control actuator and accelerometer to achieve model reference control for two test frequencies. In Sec. III B, control experiments with single and multiple control actuators are studied using the simply supported plate as the test structure.

\section{A. Simply supported beam}

The first control experiment was conducted at an excitation frequency of $300 \mathrm{~Hz}$, which is just below the second resonant frequency of the simply supported beam used in this study. The acoustic directivity pattern along the centerline of the horizontal axis of the beam resulting from the uncontrolled disturbance was initially measured in $9^{\circ}$ increments at a radius of $1.6 \mathrm{~m}$. The control algorithm was then invoked and the acoustic response of the structure was minimized at three microphone error sensors corresponding to a radius of $1.6 \mathrm{~m}$ from the beam along the horizontal centerline and at angles of $45^{\circ}, 0^{\circ}$, and $-45^{\circ}$. For convenience, $-45^{\circ}$ is used to represent the polar coordinate corresponding to $\left(\theta=45^{\circ}, \phi=180^{\circ}\right)$, while $0^{\circ}$ and $45^{\circ}$ are used to represent the polar coordinates corresponding to $\left(\theta=0^{\circ}, \phi=0^{\circ}\right)$ and $\left(\theta=45^{\circ}, \phi=0^{\circ}\right)$, respectively. A single piezoelectric actuator was used to achieve control, and the acoustic directivity pattern was again measured. Upon achieving control, a system identification was experimentally performed to compute the finite impulse response (FIR) filter necessary to create the "reference" structural response for an accelerometer located $170 \mathrm{~mm}$ from the right corner of the beam as depicted in Fig. 2. Upon determining the FIR filter required to create the "reference" plant, the model reference control approach was implemented utilizing the single accelerometer as the error sensor.

The resulting acoustic directivity patterns for the uncontrolled response, the controlled response implementing microphone error sensors and the controlled response implementing the single accelerometer error sensor are presented in Fig. 4(a). The controlled response based on mi- 


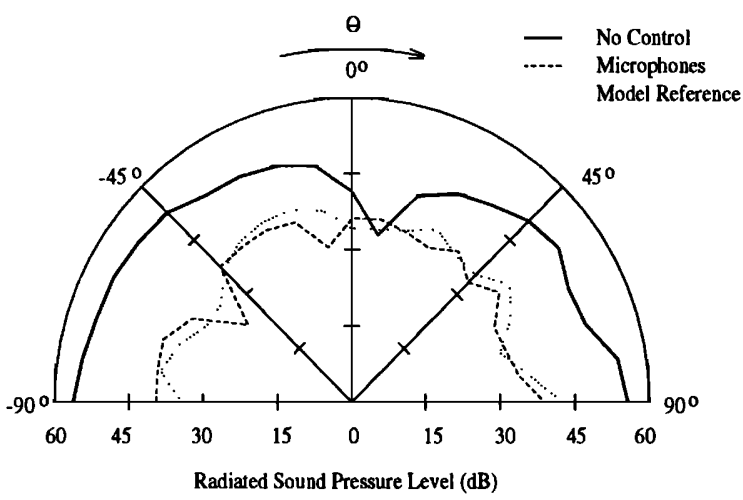

(a)

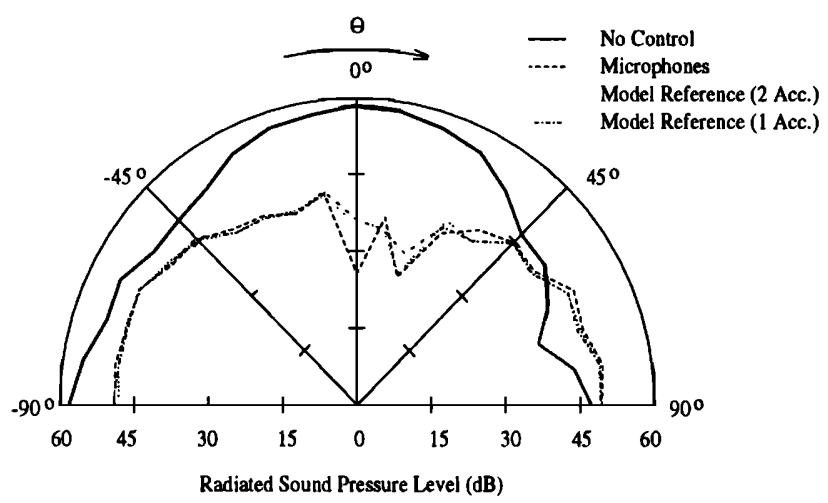

(b)

FIG. 4. Control results for simply supported beam. (a) Directivity pattern of experimental results, $f=300 \mathrm{~Hz}$. (b) Directivity pattern of experimental results, $f=660 \mathrm{~Hz}$.

crophone error sensors or model reference control with a single accelerometer resulted in sound attenuation on the order of $20 \mathrm{~dB}$ as indicated in Fig. 4(a). In addition, the directivity pattern for the model reference case closely follows that of the control case implementing microphone error sensors. One should note that while three error sensors were required to achieve the controlled acoustic directivity pattern when implementing microphones, only one accelerometer was required when performing model reference control. As discussed in previous studies, the same controlled acoustic response can be obtained if the number of model reference sensors is at least equivalent to the number of control actuators. ${ }^{7}$

The second control experiment conducted with the simply supported beam was at an excitation frequency of $660 \mathrm{~Hz}$, which is just below the third resonant frequency of the structure. As outlined for the previous test case, microphones were initially implemented as error sensors and the acoustic response at the previously outlined coordinates was minimized with a single piezoelectric control actuator. Upon achieving control, a system identification was performed to compute the necessary FIR filters for creating the "reference" plant. While only one control ac- tuator was used, tests were performed implementing two accelerometers as error sensors and then a single accelerometer as an error sensor in the model reference control approach. The second accelerometer was positioned 270 $\mathrm{mm}$ from the right of the beam as depicted in Fig. 2. These tests were performed to demonstrate that increasing the number of model reference sensors (i.e., accelerometers) beyond the number of control actuators yields no improvement in the resulting controlled response.

The acoustic directivity patterns for the three controlled responses and the uncontrolled response are presented in Fig. 4(b). As illustrated, as much as $30 \mathrm{~dB}$ of sound attenuation was achieved between $\theta=-45^{\circ}$ and $\theta=45^{\circ}$, while very little attenuation was achieved between $\theta=-45^{\circ}$ and $\theta=-90^{\circ}$ and an increase of $10 \mathrm{~dB}$ was observed between $\theta=45^{\circ}$ and $\theta=90^{\circ}$. The important observation concerns the directivity pattern for the three controlled responses. In both model reference control cases, a nearly identical acoustic directivity pattern was obtained whether one accelerometer or two accelerometers were utilized as error sensors. In addition, the acoustic directivity patterns resulting from model reference control consistently matched that resulting from control with microphone error sensors. Again this emphasizes the point made in the previous analytical study that the number of error sensors required to obtain the desired acoustic response must simply equal the number of control actuators. ${ }^{7}$

\section{B. Simply supported plate}

For the simply supported plate, two control experiments are reviewed. In the first case, the excitation frequency was chosen at $320 \mathrm{~Hz}$ which lies between the $(1,2)$ and $(2,2)$ mode of the plate used in this study. An accelerometer located at spatial coordinates of $(120,240) \mathrm{mm}$ from the lower left corner of the plate illustrated in Fig. 3 was used as an error sensor. The control actuator was centered about the spatial coordinates of $(63,150) \mathrm{mm}$ with dimensions documented in Sec. II. The acoustic response of the plate was initially controlled based on microphone error sensors located at the same coordinates as those used in the beam experiments. Upon achieving control, a system identification was performed to determine the appropriate reference plant for the model reference controller. As indicated in the plot of the measured acoustic directivity patterns in Fig. 5(a), approximately $10 \mathrm{~dB}$ of attenuation was achieved and the directivity patterns for the controlled acoustic response and the model reference response are very similar. For comparison, the model reference controller was disabled, and the response of the accelerometer was driven to zero as opposed to the desired response for controlling the sound radiation [denoted accelerometer in Fig. 5(a)]. As indicated negligible acoustic attenuation was achieved when the accelerometer output was driven to zero, emphasizing that single-point vibration control is inappropriate for controlling far-field sound radiation.

In the second control experiment conducted with the simply supported plate, the excitation frequency was set at $400 \mathrm{~Hz}$. A multiple channel control approach was taken 


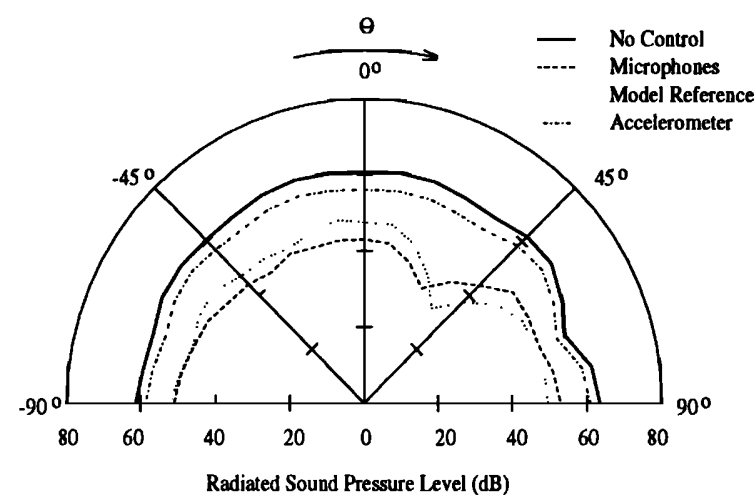

(a)

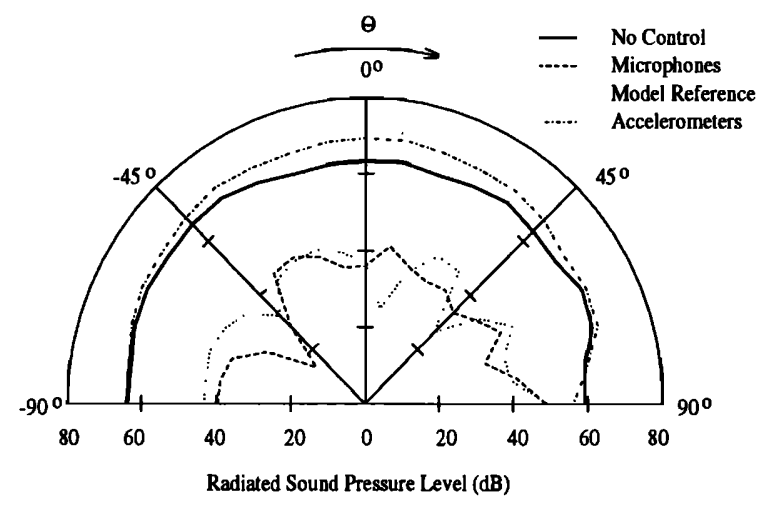

(b)

FIG. 5. Control results for simply supported plate. (a) Directivity pattern of experimental results, $f=320 \mathrm{~Hz}$. (b) Directivity pattern of experimental results, $f=400 \mathrm{~Hz}$.

with the three piezoelectric actuators located at coordinates outlined in Sec. II. Two more accelerometers were placed on the structure at spatial coordinates of $(180,200)$ $\mathrm{mm}$ and $(280,80) \mathrm{mm}$ from the lower left corner of the plate depicted in Fig. 3. Since three control inputs were used, a minimum of three accelerometers were required for the model reference control approach. The measured acoustic directivity patterns for the controlled and uncontrolled responses are presented in Fig. 5(b), and as observed, approximately $20 \mathrm{~dB}$ of acoustic attenuation was obtained when minimizing the response of the microphone error sensors or implementing the equivalent model reference control with accelerometers. The directivity patterns compare well, although some deviation is observed in the level of sound attenuation. As discussed previously in the analytical study, this error is likely attributed to inaccuracies in estimating the required reference response to achieve the desired acoustic directivity pattern. ${ }^{7}$ For comparison, the response at each accelerometer was driven to zero as opposed to the desired response, and as observed the resulting acoustic response of the structure increased by an average of $2 \mathrm{~dB}$. In general, minimizing the structural response at arbitrary coordinates will yield little if any sound attenuation unless the structure is being driven on-resonance for a given structural mode. ${ }^{7}$ Results from this test case also support the conclusion from the previous analytical study, ${ }^{7}$ suggesting that equivalent acoustic control can be achieved with structural sensors by model reference control approaches as long as the number of structural sensors is at least equal to the number of control actuators.

\section{CONCLUSIONS}

Experiments for controlling sound radiation from both a simply supported beam and plate were conducted for harmonic excitation. Results from both structures support conclusions drawn in a previous analytical study concerning advantages afforded with model reference control. ${ }^{7}$ In general, the same controlled acoustic directivity pattern can be achieved with structural error sensors as opposed to acoustic error sensors by driving the response of the structure at the sensors to a reference structural response resulting in the desired acoustic response. In addition, regardless of the number of acoustic error sensors implemented in the original control cost function, the number of structural sensors required to achieve the same controlled acoustic response must simply equal the number of control actuators used in achieving model reference control.

Disadvantages of model reference control are an increase in the $a$ priori system modeling or system identification required as well as a decrease in the flexibility afforded by the LMS algorithm. To implement the model reference control approach, the designer must either model the system in advance or experimentally determine the required reference response at chosen coordinates. Thus, accuracy of the system reference model becomes an important issue. In the previous analytical study, the sensitivity of the controlled response to errors in the reference model was studied. ${ }^{7}$ Even with errors in both the phase and magnitude of the reference models, significant acoustic attenuation was predicted, which emphasizes the fact that model reference control is based on simple physical principles. Errors in the "reference" model result in a controlled acoustic directivity pattern which is different from the desired acoustic response; however, significant sound attenuation is achieved since the relative phase referenced response of the structure at each accelerometer coordinate is physically most important for achieving the desired acoustic response. ${ }^{7}$ Experimental results from this work support this observation since the exact directivity pattern was not always achieved with model reference control; however, the level of sound attenuation was of the same order of magnitude. Model reference control simply allows the designer to impose a modal response over the surface of the structure which corresponds to the desired acoustic response.

While model reference control as implied in the name requires a system model for the desired response, the feedforward implementation ensures that the transfer function between the noise disturbance and the error sensors, in contrast to feedback methods, need not be considered. The control approach is thus relatively simple to implement on 
simple or complex structures. As with most alternative design approaches, increased design flexibility over one regime results in more restrictive limitations in others. In the case of model reference control as implemented in this study, replacing acoustic error sensors in favor of structural sensors prevents the controller from compensating for changes in the dynamics of the acoustic field once the system model is chosen. However, control of far-field sound radiation from the structure can be achieved under conditions where permanently locating microphone error sensors in the acoustic field is not possible. In spite of limitations discussed, many physical systems are based on stationary signals for which the acoustic field remains relatively unchanged, and for these cases, model reference control provides a unique method of achieving the desired acoustic response without implementing microphones as error sensors.

\section{ACKNOWLEDGMENT}

This work was supported by DARPA/ONR under Grant ONR-N00014-88-K-0721.

${ }^{1}$ R. L. Clark and C. R. Fuller, "Control of sound radiation with adaptive structures," J. Intelligent Mater. Systems Structures 2, 431-452 (1991).

${ }^{2}$ R. L. Clark and C. R. Fuller, "Modal sensing of efficient acoustic radiators with PVDF distributed sensors in active structural acoustic approaches," J. Acoust. Soc. Am. 91, 3321-3329 (1992).

${ }^{3}$ R. L. Clark and C. R. Fuller, "Active structural acoustic control with adaptive structures including wavenumber considerations," Recent Advances in Active Control of Sound and Vibration, edited by C. A. Rogers and C. R. Fuller (Technomic, Lancaster, PA, 1991), pp. 507-524.

${ }^{4}$ R. L. Clark, R. A. Burdisso, and C. R. Fuller, "Design approaches for shaping polyvinylidene fluoride sensors in active structural acoustic control (ASAC)," edited by C. A. Rogers and R. C. Rogers, proceedings of the conference on Recent Advances in Adaptive and Sensory Materials and their Applications (Technomic, Lancaster, PA, 1992), pp. 702-726.

${ }^{5}$ R. L. Clark, C. R. Fuller, B. R. Fogg, W. V. Miller, A. M. Vengsarkar, and R. O. Claus, "Structural acoustic control using optical fiber sensors and piezoelectric actuators," proceedings of Conference on Active $\mathbf{M a}$ terials and Adaptive Structures, Alexandria, VA, 5-7 November (Institute of Physics, London, 1991).

${ }^{6}$ B. R. Fogg, W. V. Miller, A. M. Vengsarkar, R. O. Claus, R. L. Clark, and C. R. Fuller "Optical fiber sensors for active structural acoustic control," Opt. Eng. 31(1) (1992).

${ }^{7}$ R. L. Clark and C. R. Fuller, "A model reference approach for implementing active structural acoustic control," J. Acoust. Soc. Am. 92, 1521-1533 (1992).

${ }^{8}$ S. J. Elliott, I. M. Strothers, and P. A. Nelson, "A multiple error LMS algorithm and its application to the active control of sound and vibration," IEEE Trans. Acoust. Speech Signal Process. ASSP-35, 1:14231434 (1987).

${ }^{9}$ J. Ochs and J. Snowdon, "Transmissibility Across Simply Supported Plates with and without Damping Layers," J. Acoust. Soc. Am. 58, 832-840 (1975).

${ }^{10} \mathrm{E}$. F. Crawley and J. de Luis, "Use of piezoelectric actuators as elements of intelligent structures," AIAA J. 25, 1373-1385 (1987).

${ }^{11}$ E. K. Dimitriadis, C. R. Fuller, and C. A. Rogers, "Piezoelectric actuators for distributed vibration excitation of thin plates," J. Vibration Acoust. 113(1), 100-107 (1991).

${ }^{12}$ R. L. Clark, C. R. Fuller, and A. L. Wicks, "Characterization of multiple Piezoceramic Actuators for Structural Excitation," J. Acoust. Soc. Am. 90, 346-357 (1991).

${ }^{13} \mathrm{~W}$. Hoppman and J. Greenspon, "An experimental device for obtaining elastic rotational constraints on the boundary of the plate," Proceedings of the 2nd National Congress on Applied Mechanics, pp. 187-191, 